\title{
A Case of Chest Pain, Abnormal Electrocardiogram and Skin Lesions
}

\author{
Yasir Parviz ${ }^{\mathrm{a}, \mathrm{c}}$, Nisar H Shah ${ }^{\mathrm{b}}$
}

\begin{abstract}
Chest pain is common presentation in emergency department. Cardiopulmonary emergencies are common and most feared but occasionally uncommon conditions may be overlooked and misdiagnosed. We report a case that presented as chest pain and left bundle branch block (LBBB) on ECG and was misdiagnosed as acute myocardial infarction. This patient was later on diagnosed with herpes zoster. This case stresses importance of thorough clinical evaluation and accurate diagnosis which helps avoid administration of unnecessary drugs with potentially serious side effects.
\end{abstract}

Keywords: Chest pain; LBBB; Herpes zoster; Thrombolysis

\section{Introduction}

Chest pain could result from variety of causes [1] ranging from relatively benign muscular pain to catastrophic emergencies. Cardiopulmonary conditions are relatively common and most feared due to their life threatening potential. Atypical presentations and uncommon conditions [1] do exist and can pose a diagnostic challenge.

We report a case of chest pain and abnormal ECG which later on developed skin lesions.

\section{Case History}

A 76-year-old, Caucasian male presented to accident and emergency department with 2 days history of dull, central

\footnotetext{
Manuscript accepted for publication July 10, 2012

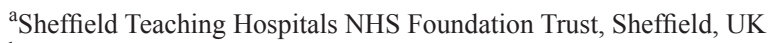

${ }^{\mathrm{b}}$ Monklands District Hospital, Airdrie, Scotland, UK

${ }^{\mathrm{c} C}$ Corresponding author: Yasir Parviz. Email: dr1yasir@hotmail.com

doi: http://dx.doi.org/10.4021/jmc803w
}

chest pain radiating to left arm, not relieved by usual analgesics. This had intensified in last few hours prior to hospitalisation. His modifiable risk factors for coronary artery disease included hypertension and hypercholesterolemia.

On initial assessment, he was still in pain, alert, apyrexial and well perfused peripherally with an unremarkable cardiac and chest examination. There was no obvious skin abnormality.

Twelve-lead ECG (Fig. 1) revealed Left bundle branch block (LBBB). There were no previous ECG's for comparison. With suggestive history and abnormal ECG an initial diagnosis of acute myocardial infarction was made. He received narcotic analgesia and was thrombolysed with reteplase. He was transferred to coronary care unit (CCU) for continued care. On admission to CCU, patient's chest pain has almost subsided.

Next morning, his cardiac enzymes (CK, Troponin T) were within the normal limits. Later on during the day, he had further episode of chest pain radiating to left arm. On examination, he was pyrexial $\left(38.6^{\circ} \mathrm{C}\right)$ with wide spread erythematous rash on left hand without neurovascular deficit or lymph adenopathy.

Few hours later his skin became itchy and developed into classical vesicular bullous appearance on forearm and hand involving C8-T1 distribution (Fig. 2a, b).

Dermatologist opinion was sought and diagnosis of herpes zoster made. Patient was prescribed Acyclovir and discharged home on day3. On follow up with dermatologist, skin lesions were healing and he was free from pain.

\section{Discussion}

Chest pain is one of the common presentations in the accident and emergency department. Uncommon conditions presenting as chest pain can, sometime, be overlooked especially if there are confounding co-morbidities [2].

Herpes Zoster (shingles) is often associated with severe pain which can precede skin eruptions for up to few days [3]. Thoracic dermatome is most common site of involvement followed by trigeminal, cervical, lumbar and sacral [4]. Shingles involving thoracic dermatomes is a differential di- 


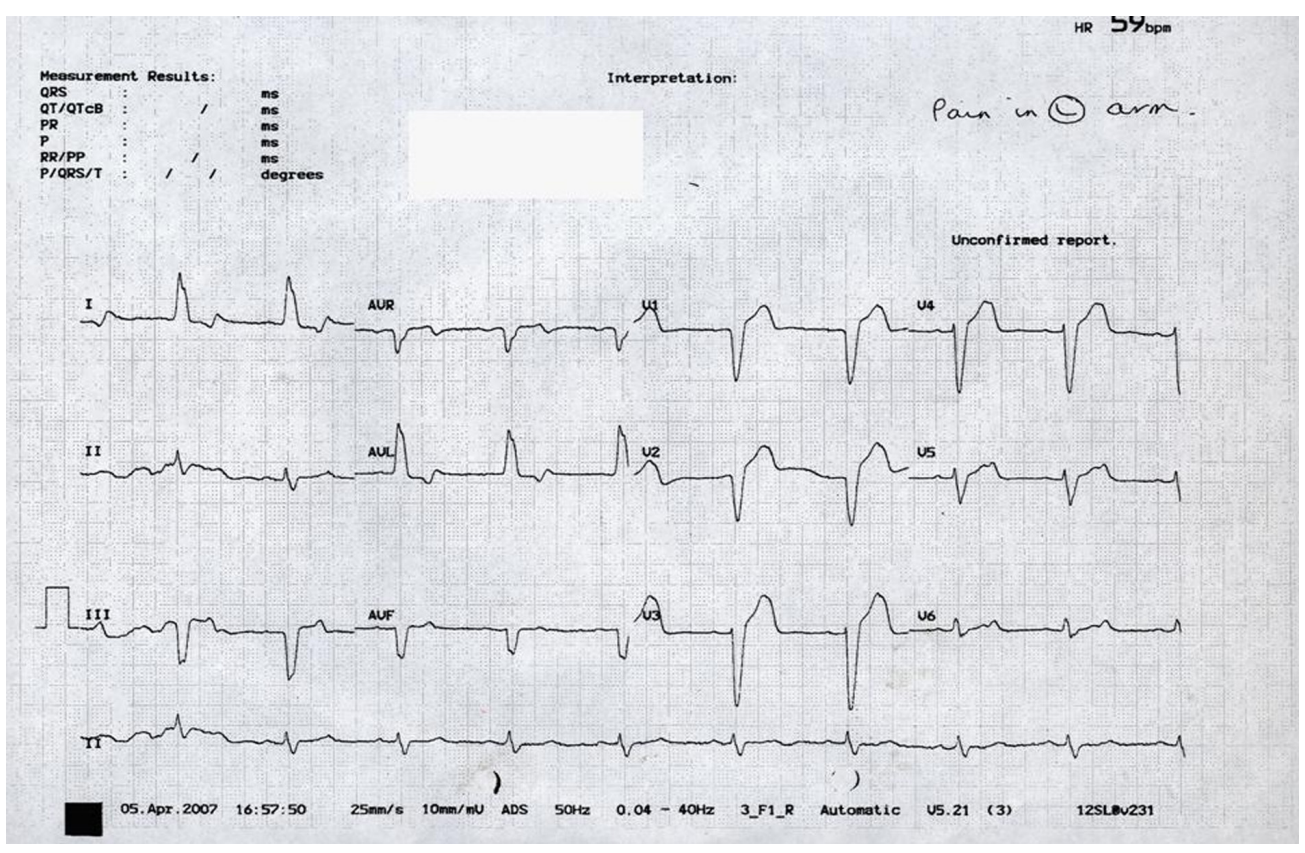

Figure 1. ECG showing left Bundle Branch Block.

agnosis of chest pain. In absence of skin lesions and atypical history, it may present a diagnostic challenge and be potentially misdiagnosed as medical or surgical emergency [5].

Ramazan Ozdemir et al [6] and Yousuf A [2] have reported cases of shingles presenting as chest pain and misdiagnosed and treated as angina pectoris. Fruergaard and colleagues [1] studied 204 patients who presented with acute chest pain without a diagnosis of myocardial infarction. Herpes zoster was diagnosed in only 1 in their series.

The case we report is unusual in certain ways. Our patient did have coronary disease risk factors, history of chest pain and abnormal ECG which all point towards more common acute coronary event. This in fact was compounded by the absence of skin abnormality on initial assessment. $\mathrm{He}$ fulfilled the criteria and received thrombolysis. Interestingly, his pain subsided with narcotic analgesics with a false impression of optimum reperfusion with thrombolysis. The diagnosis was not questioned until recurrence of chest pain and skin lesions.

This case underscores the importance of thorough clinical evaluation. Correct diagnosis leads to correct treatment and can avoid administration of medications with potentially serious side effects.

\section{Grant}

No grant involved.
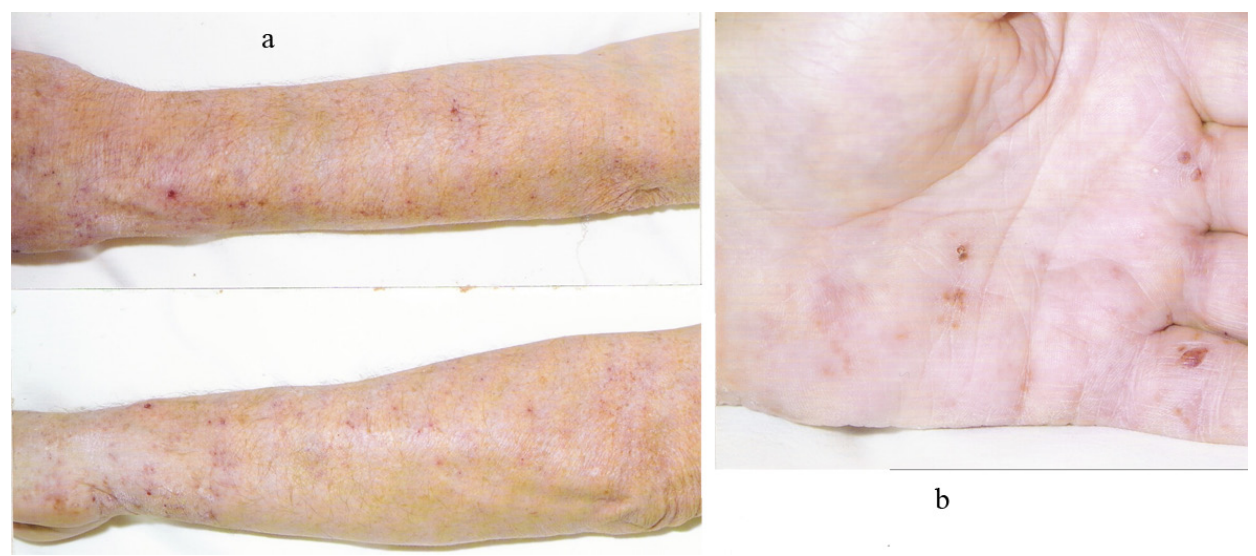

Figure 2. (a) Herpetic Vesicles on forearm; (b) Herpetic Vesicles on hand. 


\section{Conflict of Interest}

Both Authors report no conflict of interest.

\section{References}

1. Fruergaard P, Launbjerg J, Hesse B, Jorgensen F, Petri A, Eiken P, Aggestrup S, et al. The diagnoses of patients admitted with acute chest pain but without myocardial infarction. Eur Heart J. 1996;17(7):1028-1034.

2. Yousuf A. Shingles presenting as chest pain: Medicine
Today; Vol. No. 5, 4 Oct- $\quad$ Dec. 2007.

3. Reuler JB. Herpes zoster. West J Med. 1978;129(6):465-468.

4. Burgoon CF, Jr., Burgoon JS, Baldridge GD. The natural history of herpes zoster. J Am Med Assoc. 1957;164(3):265-269.

5. Morgan R, King D. Characteristics of patients with shingles admitted to a district general hospital. Postgrad Med J. 1998;74(868):101-103.

6. Ozdemir R, Tuncer C, Guven A, Sezgin AT. A case of herpes zoster misdiagnosed and treated as unstable angina pectoris. J Eur Acad Dermatol Venereol. 2000;14(4):317-319. 